\title{
Transit Photometry of the Core\#dominated Planet HD 149026b
}

\section{Citation}

Charbonneau, David, Joshua N. Winn, David W. Latham, Gaspar Bakos, Emilio E. Falco, Matthew J. Holman, Robert W. Noyes, et al. 2006. "Transit Photometry of the Core\#dominated Planet HD 149026b." The Astrophysical Journal 636 (1): 445-52. https://doi.org/10.1086/497959.

\section{Permanent link}

http://nrs.harvard.edu/urn-3:HUL.InstRepos:41397427

\section{Terms of Use}

This article was downloaded from Harvard University's DASH repository, and is made available under the terms and conditions applicable to Other Posted Material, as set forth at http:// nrs.harvard.edu/urn-3:HUL.InstRepos:dash.current.terms-of-use\#LAA

\section{Share Your Story}

The Harvard community has made this article openly available.

Please share how this access benefits you. Submit a story.

Accessibility 


\title{
TRANSIT PHOTOMETRY OF THE CORE-DOMINATED PLANET HD 149026b
}

\author{
David Charbonneau, Joshua N. Winn, ${ }^{1}$ David W. Latham, Gáspár Bakos, ${ }^{1}$ Emilio E. Falco, \\ Matthew J. Holman, Robert W. Noyes, and Balázs Csák ${ }^{2}$ \\ Harvard-Smithsonian Center for Astrophysics, 60 Garden Street, Cambridge, MA 02138; dcharbonneau@cfa.harvard.edu
}

Gilbert A. Esquerdo and Mark E. Everett

Planetary Science Institute, 1700 East Fort Lowell, Tucson, AZ 85719

AND

Francis T. O'Donovan

California Institute of Technology, 1200 East California Boulevard, Pasadena, CA 91125

Received 2005 August 1; accepted 2005 September 8

\begin{abstract}
We report $g, V$, and $r$ photometric time series of HD 149026 spanning predicted times of transit of the Saturn-mass planetary companion, which was recently discovered by Sato and collaborators. We present a joint analysis of our observations and the previously reported photometry and radial velocities of the central star. We refine the estimate of the transit ephemeris to $T_{c}=\left(2,453,527.87455_{-0.00091}^{+0.00085}\right)+\left(2.87598_{-0.00017}^{+0.00012}\right) N($ HJD). Assuming that the star has a radius of $1.45 \pm 0.10 R_{\odot}$ and a mass of $1.30 \pm 0.10 M_{\odot}$, we estimate the planet radius to be $(0.726 \pm 0.064) R_{\text {Jup }}$, which implies a mean density of $1.07_{-030}^{+0.42} \mathrm{~g} \mathrm{~cm}^{-3}$. This density is significantly greater than predicted for models that include the effects of stellar insolation and in which the planet has only a small core of solid material. Thus, we confirm that this planet likely contains a large core and that the ratio of core mass to total planet mass is more akin to that of Uranus and Neptune than to either Jupiter or Saturn.
\end{abstract}

Subject headings: planetary systems - stars: individual (HD 149026) — techniques: photometric

Online material: machine-readable table

\section{INTRODUCTION}

Sato et al. (2005) recently presented the discovery of a planetary companion to the bright G0 IV star HD 149026. The star exhibits a time-variable Doppler shift that is consistent with a sinusoid of amplitude $K=43 \mathrm{~m} \mathrm{~s}^{-1}$ and period $\mathcal{P}=2.9$ days, which would be produced by the gravitational force from an orbiting planet with $M_{P} \sin i=0.36 M_{\mathrm{Jup}}$. Furthermore, at the predicted time of planet-star conjunction, the star's flux declines by $0.3 \%$ in the manner expected of an eclipse by a planet of radius $0.72 R_{\text {Jup }}$ (given an estimate of the stellar radius, $1.45 R_{\odot}$, which is based on the stellar parallax and effective temperature). Sato et al. (2005) observed three such eclipses. This discovery is extraordinary for at least two reasons.

First, the occurrence of eclipses admits this system into the elite club of bright stars with detectable planetary transits. Of all the previously known transiting systems, only HD 209458 (Charbonneau et al. 2000; Henry et al. 2000) and TrES-1 (Alonso et al. 2004; Sozzetti et al. 2004) have parent stars brighter than $V=12$, and therefore only they are amenable to a number of fascinating measurements requiring a very high signal-to-noise ratio. Among these studies are (1) the search for satellites and rings (Brown et al. 2001), (2) the search for period variations due to additional companions (Wittenmyer et al. 2005), (3) the detection of (or upper limits on) atmospheric absorption features in transmission (Charbonneau et al. 2002; Brown et al. 2002; Deming et al. 2005a), (4) the characterization of the exosphere

\footnotetext{
1 Hubble Fellow.

2 SAO Predoctoral Fellow. Home institution: Department of Experimental Physics and Astronomical Observatory, University of Szeged, Dóm tér 9, 6720 Szeged, Hungary.
}

(Bundy \& Marcy 2000; Moutou et al. 2001, 2003; Vidal-Madjar et al. 2003, 2004; Winn et al. 2004; Narita et al. 2005), (5) the measurement of the angle between the sky-projected orbit normal and stellar rotation axis (Queloz et al. 2000; Winn et al. 2005), (6) the search for spectroscopic features near the times of secondary eclipse (Richardson et al. 2003a, 2003b), and (7) the direct detection of thermal emission from the planet (Charbonneau et al. 2005; Deming et al. 2005b). Charbonneau (2004) reviewed these techniques and related investigations.

Second, the planet is the smallest and least massive of the eight known transiting extrasolar planets. ${ }^{3}$ This makes HD $149026 \mathrm{~b}$ an important test case for theories of planetary structure. Sato et al. (2005) argued that, once the effects of stellar insolation are included, the small planetary radius implies that the planet has a large and dense core. In particular, assuming a core density $\rho_{c}=5.5 \mathrm{~g} \mathrm{~cm}^{-3}$, their model demands a prodigious core mass of 78 Earth masses, or $74 \%$ of the total mass of the planet. This, in turn, would seemingly prove that the planet formed through core accretion, as opposed to direct collapse through a gravitational instability.

A system of such importance should be independently confirmed, and the determination of its basic parameters should be refined through multiple observations. With this as motivation, we performed photometry of HD 149026 on two different nights when transits were predicted by Sato et al. (2005). These observations and the data reduction procedures are described in $\S 2$.

\footnotetext{
${ }^{3}$ In addition to HD 209458b and TrES-1, the OGLE photometric survey (Udalski et al. 2002, 2004) and spectroscopic follow-up efforts have located five such objects. Recent estimates of the planetary radii have been given by Bouchy et al. (2004), Holman et al. (2006), Konacki et al. (2003, 2005), Moutou et al. (2004), and Pont et al. (2004) and references therein.
} 
The model that we used to determine the system parameters is described in $\S 3$, and the results are discussed in $\S 4$. Our data are available in digital form in the electronic version of this article and from the authors upon request.

\section{THE OBSERVATIONS AND DATA REDUCTION}

\subsection{FLWO $1.2 \mathrm{~m} g$ and $r$ Photometry}

We observed HD $149026(V=8.16, B-V=0.56)$ on UT 2005 June 6 and July 2 with the 48 inch $(1.2 \mathrm{~m})$ telescope of the F. L. Whipple Observatory (FLWO) located at Mount Hopkins, Arizona. We used Minicam, an optical CCD imager with two $2048 \times 4608$ chips. In order to increase the duty cycle of the observations, we employed $2 \times 2$ binning, which reduced the readout and overhead time to $20 \mathrm{~s}$. Each binned pixel subtends approximately $0 .{ }^{\prime \prime} 6$ on the sky, giving an effective field of view of about $10^{\prime} \times 23^{\prime}$ for each CCD. Fortunately, there exists a nearby object of similar brightness and color (HD 149083; $V=8.05$, $B-V=0.40, \Delta \alpha=5$ '. $\left.^{\prime}, \Delta \delta=-17^{\prime}\right)$, which we employed as an extinction calibrator. We selected the telescope pointing so that both stars were imaged simultaneously. We defocused the telescope so that the FWHM of a stellar image was typically 15 binned pixels $\left(9^{\prime \prime}\right)$, and we used automatic guiding to ensure that the centroid of the stellar images drifted no more than 3 binned pixels over the course of the night. In addition to enabling longer integration times, this served to mitigate the effects of pixel-to-pixel sensitivity variations that were not perfectly corrected by our flat-fielding procedure. On UT 2005 June 6, we gathered $5.5 \mathrm{hr}$ of Sloan Digital Sky Survey (SDSS) $g$-band observations with typical integration times of $8 \mathrm{~s}$ and a cadence of $28 \mathrm{~s}$. The conditions were photometric, and the frames span an air mass from 1.01 to 1.74 . On UT 2005 July 2 , we gathered $4.4 \mathrm{hr}$ of SDSS $r$-band observations with integration times of $6 \mathrm{~s}$ and a median cadence of $26 \mathrm{~s}$. The field appeared to remain free of clouds for the duration of the observations, which spanned an air mass range of 1.01 to 1.43 , although occasional patches of high cirrus could be seen in images from the MMT all-sky camera.

We converted the image time stamps to Heliocentric Julian Date (HJD) at mid-exposure. The images were overscan-subtracted, trimmed, and divided by a flat-field image. We performed skysubtracted aperture photometry of HD 149026 and the comparison star HD 149083. The details of the analysis differed slightly for the two nights. For the UT 2005 June 6 data, we used an aperture radius of 15 binned pixels $\left(9^{\prime \prime}\right)$. We estimated the contribution from the sky by using the IRAF APPHOT software package with an inner radius of 150 pixels and an outer radius of 180 pixels, computing the median value and the standard deviation $\sigma$, rejecting $3 \sigma$ outliers, and iterating this process until no such outliers remained. Our estimate of the sky counts per pixel for each star was the final median value calculated in this manner. For the UT 2005 July 2 data, we used an aperture radius of 20 binned pixels $\left(12^{\prime \prime}\right)$. To measure the background sky flux, we extracted pixel values from an annulus centered on each star having an inner radius of 40 and an outer radius of 90 binned pixels. We calculated the standard deviation $\sigma$ and rejected pixel values deviating by more than $3 \sigma$ from the mode. We then iterated this process 10 times, or until no more pixels were rejected. We took the final modal pixel value as the sky flux per pixel for each star. Both of the methods described above provide robust estimates of the sky contribution, and we note that neither sky annulus contained a star of sufficient brightness to affect our estimate of the transit depth. For each night, we then computed the relative flux of HD 149026 by taking the ratio of the fluxes within the two sky-subtracted apertures. Normalization and residual extinction corrections are described in $\S 3$.

\subsection{TopHAT V Photometry at FLWO}

TopHAT is an automated telescope located at Mt. Hopkins, Arizona, which was designed to perform multicolor photometric follow-up of transiting extrasolar planet candidates identified by the HAT network (Bakos et al. 2004). Since TopHAT has not previously been described in the literature, we digress briefly to outline the principal goals and features of the instrument.

Wide-field transit surveys must contend with a large rate of astrophysical false positives, which result from stellar systems that contain an eclipsing binary and precisely mimic the singlecolor photometric light curve of a Jupiter-sized planet transiting a Sun-like star (Brown 2003; Charbonneau et al. 2004; Mandushev et al. 2005; Torres et al. 2004). Although multiepoch radial velocity follow-up is an effective tool for identifying these false positives (e.g., Latham 2003), instruments such as TopHAT and Sherlock (Kotredes et al. 2004) can be fully automated and thus offer a very efficient means of culling the bulk of such false positives. TopHAT is a $0.26 \mathrm{~m}$ diameter $\mathrm{f} / 5$ commercially available Baker Ritchey-Chrétien telescope on an equatorial fork mount developed by Fornax Inc. A 1.25 square field of view is imaged onto a $2 \mathrm{k} \times 2 \mathrm{k}$ Peltier-cooled, thinned CCD detector, yielding a pixel scale of 2".2. The time for image readout and associated overheads is $25 \mathrm{~s}$. Well-focused images have a typical FWHM of 2 pixels. A two-slot filter exchanger permits imaging in either $V$ or $I$. The components are protected from inclement weather by an automated asymmetric clamshell dome.

We observed HD 149026 on UT 2005 July 2, the same night as the FLWO $1.2 \mathrm{~m} r$ observations described above. In order to extend the integration times and increase the duty cycle of the observations, we broadened the point-spread function (PSF) by performing small, regular motions in right ascension and declination according to a prescribed pattern that was repeated during each $13 \mathrm{~s}$ integration (see Bakos et al. 2004 for details). The resulting PSF had a FWHM of 3.5 pixels (7.7). We gathered $4.8 \mathrm{hr}$ of $V$ observations with a cadence of $68 \mathrm{~s}$, spanning an air mass range of 1.01 to 1.45 .

We converted the time stamps in the image headers to HJD at mid-exposure. We calibrated the images by subtracting the overscan bias and a scaled dark image, and dividing by an average sky flat from which large outliers had been rejected. We evaluated the centroids of the target and the three brightest calibrators in each image. For each star, we summed the flux within an aperture with a radius of 8 pixels (17.'6) and performed a sky subtraction. We estimated the contribution from the sky in an annulus surrounding each photometric aperture using the algorithm adopted by DAOPHOT II (e.g., Stetson 1994), which compares the mean and median after sigma-clipping to estimate and remove contamination from stars in the sky aperture. We then divided the resulting time series for the target by the statistically weighted average of the time series for the three calibrator stars. The resulting relative flux time series was then corrected for normalization and residual extinction effects as described in $\S 3$.

\section{THE MODEL}

We attempted to fit simultaneously (1) the three photometric time series discussed above, $(2)$ the $(b+y) / 2$ photometry of three transits presented by Sato et al. (2005), and (3) the seven radial velocities that were measured by Sato et al. (2005) when the planet was not transiting. We did not attempt to fit the four radial 

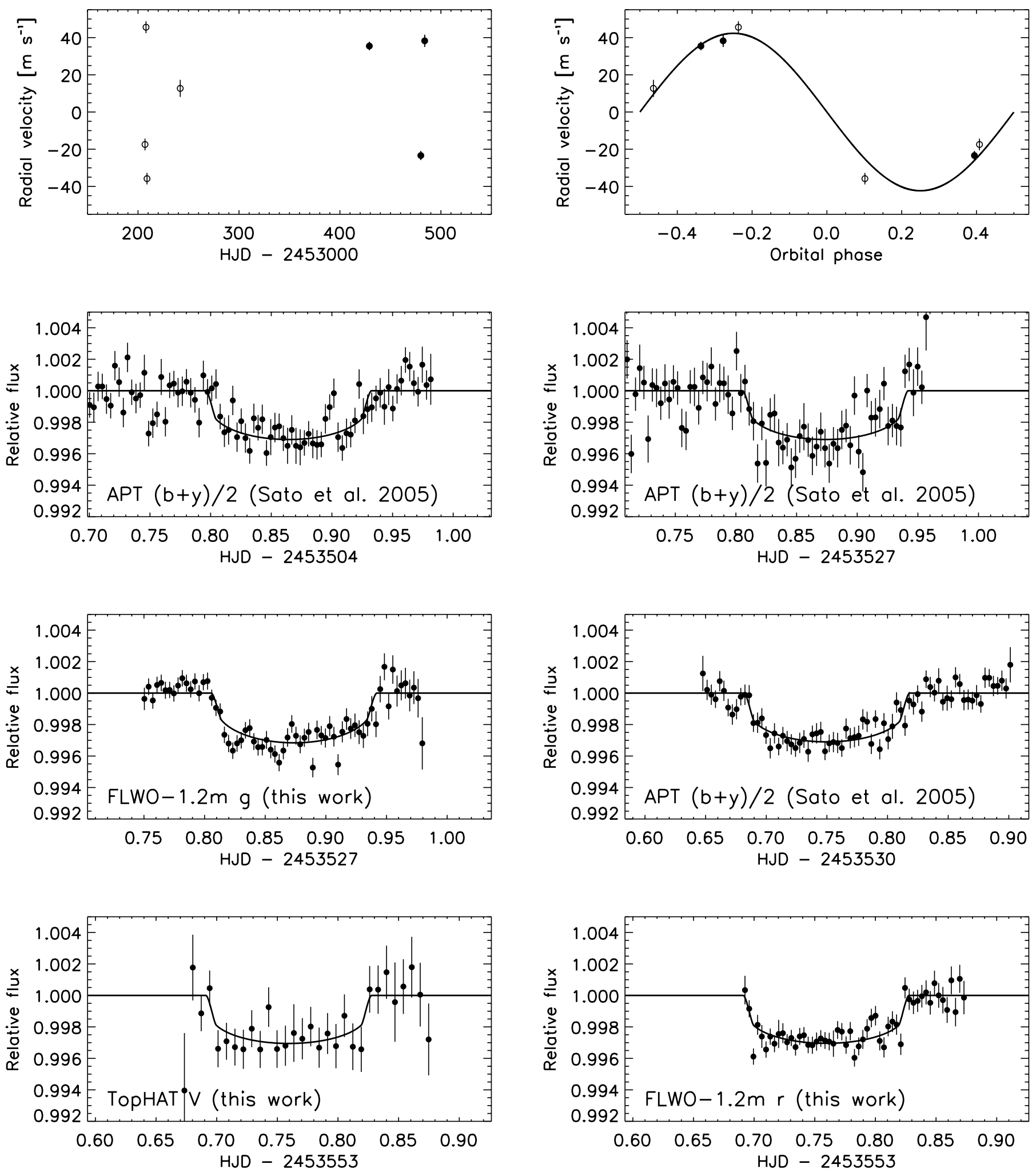

FIG. 1.-Radial velocity variations and photometry of HD 149026. The top two panels show the radial velocity measurements by Sato et al. (2005), as a function of time (left) and orbital phase (right). Open circles are Subaru HDS measurements, and filled circles are Keck HIRES measurements. The best-fitting value of $\gamma$ (for each spectrograph) has been subtracted from the data. The best-fitting model is overplotted. The remaining panels show the photometry from this work and Sato et al. (2005). Although the points have been averaged into 5 minute bins for presentation purposes (10 minute bins for TopHAT), the fitting procedure was performed on unbinned data. 
velocities measured during transits, which would have required a model of the Rossiter-McLaughlin effect (Queloz et al. 2000; Ohta et al. 2005; Winn et al. 2005).

We modeled the system as a circular Keplerian orbit. Following Sato et al. (2005), we assumed the stellar mass $\left(M_{*}\right)$ to be $1.30 M_{\odot}$ and the stellar radius ${ }^{4}\left(R_{*}\right)$ to be $1.45 R_{\odot}$. The free parameters were the planetary mass $\left(M_{P}\right)$, planetary radius $\left(R_{P}\right)$, orbital inclination $(i)$, orbital period $(\mathcal{P})$, central transit time $\left(T_{c}\right)$, and the heliocentric radial velocity of the center of mass $(\gamma)$. We included two free parameters for each of our three photometric time series: an overall flux scaling $C$; and a residual extinction coefficient $(k)$ to correct for differential extinction between the target star and the comparison object, which have somewhat different colors. These are defined such that the relative flux observed through an air mass $X$ is $C \exp (-k X)$ times the true relative flux. The residual extinction corrections were small but important at the millimagnitude level; for example, in the $g$ band, we found $k \approx-2 \times 10^{-3}$. The three time series presented by Sato et al. (2005) were already corrected for air mass, so we allowed each of these to have only an independent flux scaling. Finally, we allowed the data from each spectrograph (the Keck High Resolution Echelle Spectrometer [HIRES] and the Subaru High Dispersion Spectrograph [HDS]) to have an independent value of $\gamma$.

We computed the model radial velocity at each observed time as $\gamma+\Delta v_{r}$, where $\Delta v_{r}$ is the line-of-sight projection of the orbital velocity of the star. We calculated the model flux during transit using the linear limb-darkening law $B_{\lambda}(\mu)=1-u_{\lambda}(1-\mu)$, where $B_{\lambda}$ is the normalized stellar surface brightness profile and $\mu$ is the cosine of the angle between the normal to the stellar surface and the line of sight. We employed the "small planet" approximation as described by Mandel \& Agol (2002). We held the limb-darkening parameter $u_{\lambda}$ fixed at a value appropriate for a star with the assumed properties and for the bandpass concerned, according to the models of Claret \& Hauschildt (2003) and Claret (2004). These values were $u_{g}=0.73, u_{r}=0.61, u_{V}=$ 0.62 , and $u_{b+y}=0.67$.

The goodness-of-fit parameter is

$$
\chi^{2}=\chi_{v}^{2}+\chi_{f}^{2}=\sum_{n=1}^{N_{v}}\left(\frac{v_{O}-v_{C}}{\sigma_{v}}\right)^{2}+\sum_{n=1}^{N_{f}}\left(\frac{f_{O}-f_{C}}{\sigma_{f}}\right)^{2}
$$

where $v_{O}$ and $v_{C}$ are the observed and calculated radial velocities, of which there are $N_{v}=7$, and $f_{O}$ and $f_{C}$ are the observed and calculated fluxes, of which there are $N_{f}=2310$. Of the flux measurements, 679 are our $g$-band measurements, 574 are our $r$-band measurements, 237 are our $V$-band measurements, and 820 are the $(b+y) / 2$ measurements of Sato et al. (2005). We minimized $\chi^{2}$ using an AMOEBA algorithm (Press et al. 1992).

The radial velocity uncertainties $\sigma_{v}$ were taken from Table 2 of Sato et al. (2005). To estimate the uncertainties in our photometry, we performed the following procedure. We expect the two dominant sources of uncertainty to be scintillation noise $\left(\sigma_{S}\right)$ and

\footnotetext{
${ }^{4}$ With more accurate photometry and better time sampling of the ingress or egress, it would be possible to solve for the stellar radius, rather than assuming a certain value (see, e.g., Brown et al. 2001; Winn et al. 2005; Wittenmyer et al. 2005; and Holman et al. 2006). In this case, we found that the stellar radius is not well determined by the photometry. Rather, the constraint on the stellar radius based on stellar parallax and spectral modeling is tighter.
}

TABLE 1

РнотоMETRY OF HD 149026

\begin{tabular}{ccccc}
\hline \hline Telescope & Filter & HJD & Relative Flux & Uncertainty \\
\hline FLWO48........... & $g$ & 2453527.750311 & 0.9983 & 0.0018 \\
FLWO48......... & $g$ & 2453527.750624 & 0.9984 & 0.0018 \\
FLWO48.......... & $g$ & 2453527.750936 & 0.9988 & 0.0018 \\
FLWO48.......... & $g$ & 2453527.751260 & 0.9999 & 0.0018 \\
FLWO48........... & $g$ & 2453527.751585 & 1.0006 & 0.0018 \\
\hline
\end{tabular}

Notes.-The quoted uncertainties are based on the procedure described in $\S 2$, which assumes that our model is correct. Table 1 is published in its entirety in the electronic edition of the Astrophysical Journal. A portion is shown here for guidance regarding its form and content. The data are also available in digital form from the authors upon request.

Poisson noise $\left(\sigma_{\mathrm{P}}\right)$. Dravins et al. (1998) state the following expression for $\sigma_{S}$, the fractional error due to scintillation noise:

$$
\sigma_{S}=0.09 D^{-2 / 3}(\sec Z)^{1.75} \exp \left(\frac{-h}{h_{0}}\right)(2 T)^{-1 / 2},
$$

where $D$ is the diameter of the aperture in $\mathrm{cm}, \sec Z$ is the air mass, $h$ is the altitude of the observatory, $h_{0}=8000 \mathrm{~m}$ is the atmospheric scale height, and $T$ is the integration time in seconds. For each of our three time series, we assumed that the noise in each point obeyed

$$
\sigma=\sqrt{\sigma_{P}^{2}+\left(\beta \sigma_{S}\right)^{2}}
$$

where $\sigma_{S}$ was calculated with equation (2). We determined the constant $\beta$ by requiring $\chi^{2} / N_{\text {DOF }}=1$ for that particular time series. Thus we did not attempt to use the $\chi^{2}$ statistic to test the validity of the model; rather, we assumed the model was correct and sought the appropriate weight for each data point. The results for $\beta$ ranged from 1.2 to 1.5. To estimate the uncertainties in each of the three Sato et al. (2005) time series (which were already corrected for air mass), we simply assigned an air massindependent error bar to all the points such that $\chi^{2} / N_{\text {DOF }}=1$. The results agreed well with the rms values quoted in Table 5 of Sato et al. (2005).

After assigning the weight of each data point in this manner, we analyzed all the data simultaneously and found the bestfitting solution. This solution is overplotted on the data in Figure 1. Our photometry, after correcting for the overall flux scale and for air mass, is given in Table 1 . We estimated the uncertainties in the model parameters using a Monte Carlo algorithm, in which the optimization was performed on each of $7 \times 10^{4}$ synthetic data sets, and the distribution of best-fitting values was taken to be the joint probability distribution of the parameters. Each synthetic data set was created as follows:

1. We randomly drew $N_{f}=2310$ flux measurements $\left\{t_{n}, f_{n}\right\}$ from the real data set. We drew these points with replacements, i.e., we allowed for repetitions of the original data points. This procedure, recommended by Press et al. (1992), estimates the probability distribution of the measurements using the measured data values themselves, rather than the more traditional approach of assuming a certain model for the underlying process.

2 . This procedure was impractical for the radial velocity measurements, because the number of data points is too small. Instead, in each realization, we discarded a single radial velocity measurement chosen at random. This was intended as a test of the robustness of the results to single outliers. To each of the remaining 

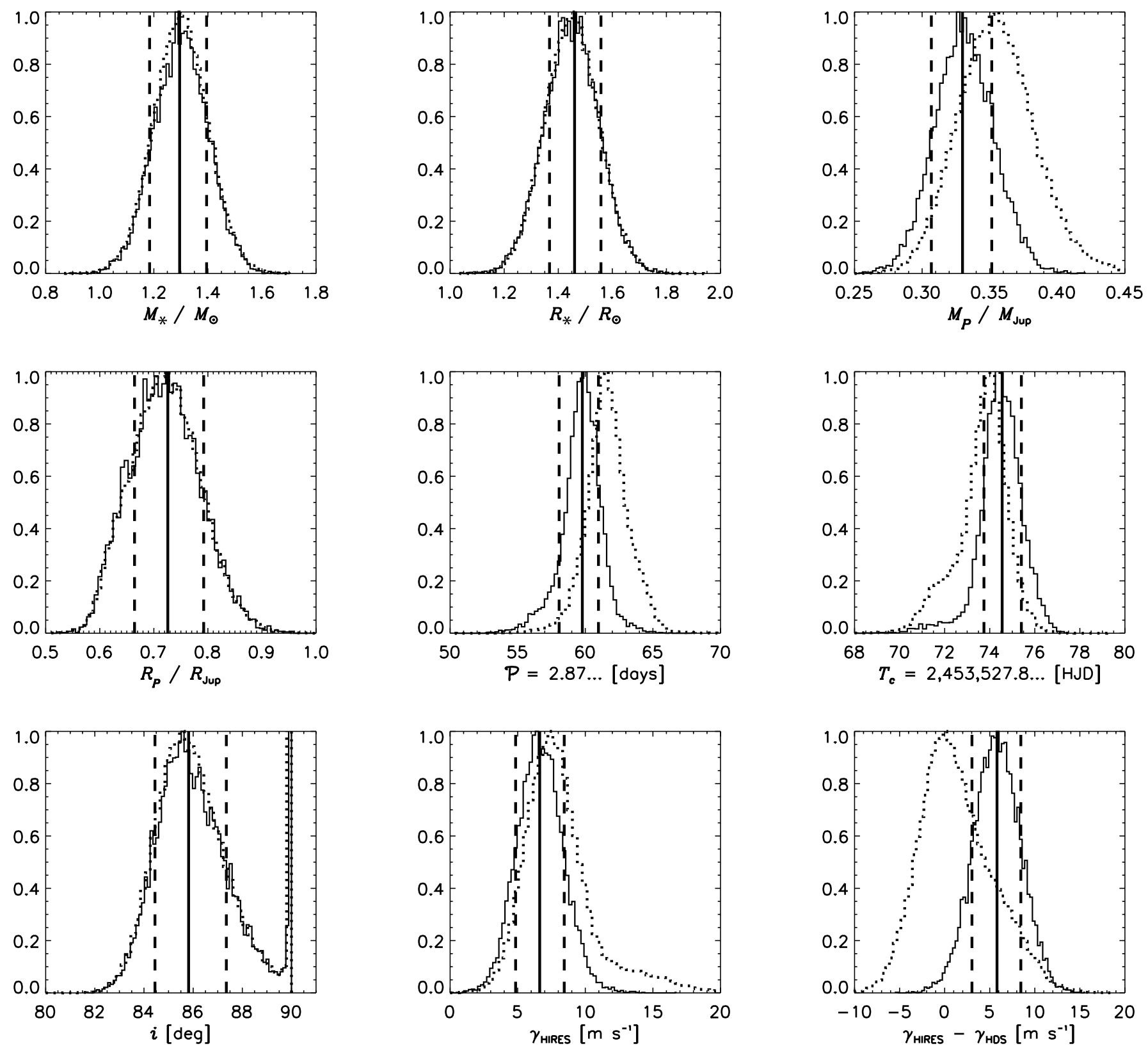

FIG. 2.-Estimated probability distributions of some planetary, stellar, and orbital parameters. The dotted histograms show the results of fits to all $7 \times 10^{4}$ Monte Carlo realizations of the data. The solid histograms show the results for the cases where the biggest radial velocity outlier is dropped; we favor these results for reasons described in the text. The one-dimensional probability distribution for $i$ (bottom left panel) shows a secondary peak near $i=90^{\circ}$, which contains $9 \%$ of the solutions. We clip these solutions prior to determining the best-fit values and confidence limits (see text). Solid vertical lines show the median value of each of the solid histograms. Dashed vertical lines show the $68.3 \%$ confidence limits.

$N_{v}=6$ radial velocities, we added a random number drawn from a Gaussian distribution, with zero mean and a standard deviation equal to the quoted $1 \sigma$ uncertainty.

3. To account for the uncertainty in the stellar properties, we assigned a stellar mass by picking a random number from a Gaussian distribution with mean $1.30 M_{\odot}$ and standard deviation $0.10 M_{\odot}$. Likewise, for the stellar radius, we used a Gaussian distribution with a mean of $1.45 R_{\odot}$ and a standard deviation of $0.10 R_{\odot}$. We note that this procedure does not take into account the intrinsic correlation between these two variables that is expected from models of stellar structure and evolution. Assuming a stellar mass-radius relation would provide an independent constraint that would reduce the overall uncertainty on the planetary radius (Cody \& Sasselov 2002). We elected not to make such an assumption because the stellar age and therefore its evolutionary state are not known with sufficient precision.

\section{DISCUSSION AND CONCLUSIONS}

The best-fitting model is illustrated in Figure 1. The estimated probability distribution for each model parameter is shown in Figure 2. Some of the parameters have correlated uncertainties, as shown in Figure 3. In addition to showing correlations among the model parameters, Figure 3 shows the distributions for three fundamental properties of a single-transit light curve: the transit depth in the absence of stellar limb darkening, defined as $\left(R_{P} / R_{*}\right)^{2}$; the transit duration, defined as the time between first and fourth contact $\left(t_{\mathrm{IV}}-t_{\mathrm{I}}\right)$; and the ingress duration, defined as the time between first and second contact $\left(t_{\mathrm{II}}-t_{\mathrm{I}}\right)$. Since we have assumed a 

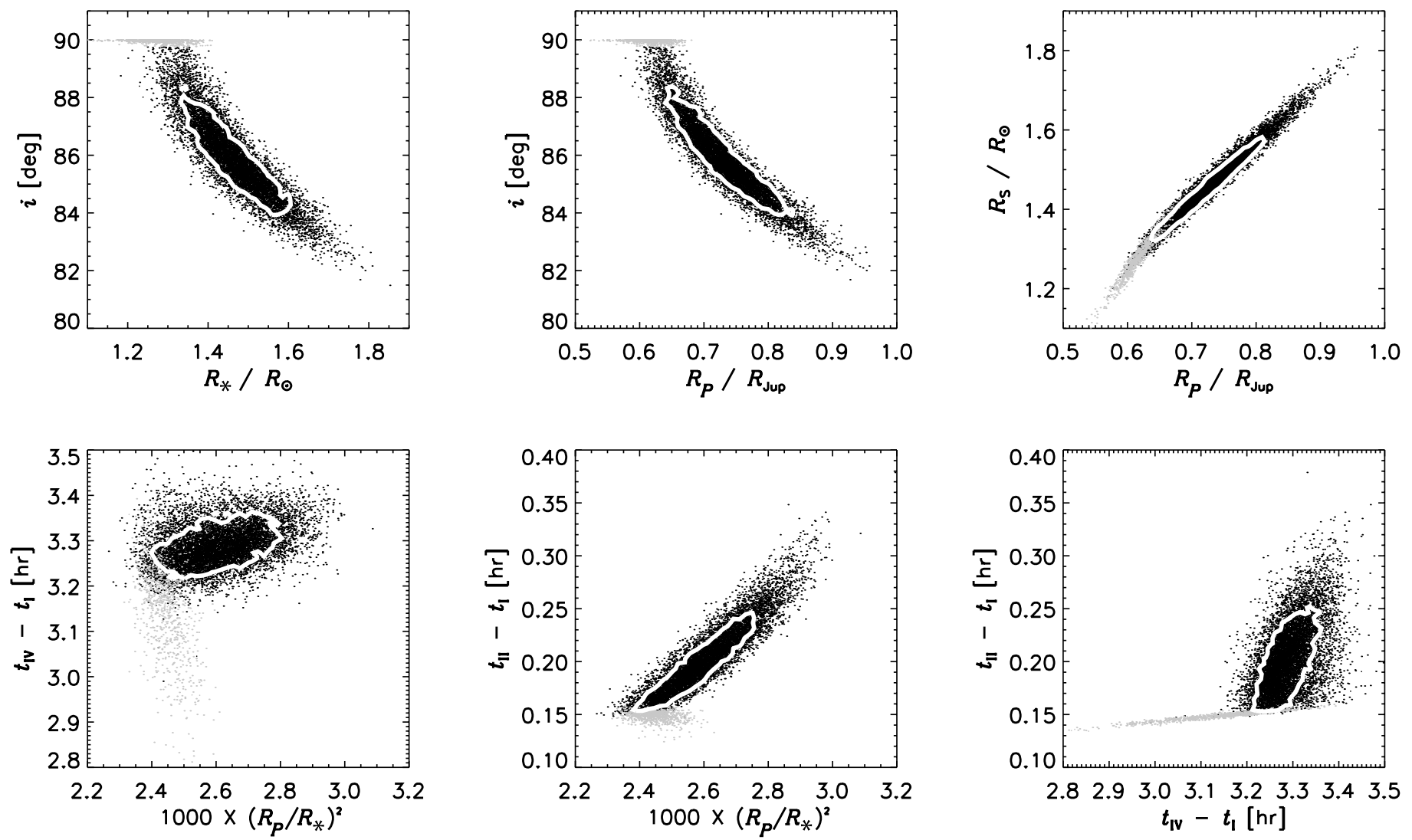

FIG. 3.- Joint probability distributions of some planetary, stellar, and orbital parameters, based on the $10^{4}$ Monte Carlo realizations in which $v_{3}$ was dropped. The

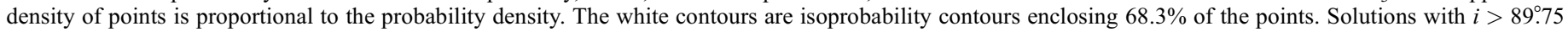
(an arbitrary threshold) are colored gray to distinguish between the two solution branches described in the text.

circular Keplerian orbit, the ingress and egress durations are equal. The contact times can be calculated from our model parameters via the relations

$$
\begin{aligned}
& \sin i \cos \frac{\pi\left(t_{\mathrm{IV}}-t_{\mathrm{I}}\right)}{\mathcal{P}}=\sqrt{1-\left(\frac{R_{*}+R_{P}}{a}\right)^{2}} \\
& \sin i \cos \frac{\pi\left(t_{\mathrm{II}}-t_{\mathrm{I}}\right)}{\mathcal{P}}=\sqrt{1-\left(\frac{R_{*}-R_{P}}{a}\right)^{2}},
\end{aligned}
$$

where $a$ is the semimajor axis, given by Newton's revised version of Kepler's third law $G\left(M_{*}+M_{P}\right) / a^{3}=(2 \pi / \mathcal{P})^{2}$.

We found that the probability distributions did not depend significantly on which of the seven radial velocity measurements were discarded, with the important exception of the third Subaru HDS measurement $\left(v_{3}=-42.48 \pm 3.10 \mathrm{~m} \mathrm{~s}^{-1}\right.$ at HJD 2,453,208.909939). When this point was dropped, the results for the orbital period, planetary mass, and velocity offsets changed by $1 \sigma$. In Figure 2, the solid histograms show the results of the $10^{4}$ trials for which $v_{3}$ was discarded, and the dotted histograms show the results of all $7 \times 10^{4}$ trials. We believe either that the model fails to accurately describe $v_{3}$ within its quoted uncertainty (due to a missing ingredient such as a nonzero eccentricity ${ }^{5}$ or an additional planetary companion), or that the true uncertainty in this measurement is larger than the estimate (due to stellar jitter or some other source of systematic error). We note

\footnotetext{
${ }^{5}$ We verified that a nonzero eccentricity is sufficient to account for $v_{3}$ within the quoted uncertainty. In the best-fitting model, $e=0.1$.
}

that this single point makes a disproportionate contribution to the $\chi_{v}^{2}$. When any single radial velocity measurement besides $v_{3}$ is dropped, the value of $\chi_{v}^{2}$ is reduced by $0.3-5$. Yet when $v_{3}$ is dropped, $\chi_{v}^{2}$ is reduced by 18 , a dramatic improvement suggesting that either the error associated with this point is underestimated or the model is inadequate. In Table 2, we present the results for the cases in which $v_{3}$ is dropped. Our intention is to avoid biased results from an oversimplified model or an underestimated uncertainty.

TABLE 2

\begin{tabular}{|c|c|c|c|}
\hline \multirow[b]{2}{*}{ PARAmeter } & \multirow[b]{2}{*}{ Best Fit } & \multicolumn{2}{|c|}{$68 \%$ Confidence Limits } \\
\hline & & Lower & Upper \\
\hline$M_{*}\left(M_{\odot}\right) \ldots \ldots \ldots \ldots$ & 1.30 & -0.10 & +0.10 \\
\hline$R_{*}\left(R_{\odot}\right)$ & 1.45 & -0.10 & +0.10 \\
\hline$M_{P}\left(M_{\mathrm{Jup}}\right)$ & 0.330 & -0.023 & +0.022 \\
\hline$R_{P}\left(R_{\mathrm{Jup}}\right) \ldots \ldots$ & 0.726 & -0.062 & +0.066 \\
\hline $\mathcal{P}$ (days) & 2.87598 & -0.00017 & +0.00012 \\
\hline 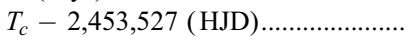 & 0.87455 & -0.00091 & +0.00085 \\
\hline$i(\mathrm{deg})$ & 85.8 & -1.3 & +1.6 \\
\hline 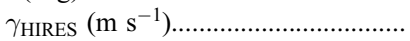 & 6.6 & -1.8 & +1.8 \\
\hline 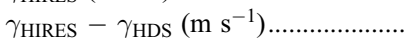 & 5.8 & -2.8 & +2.6 \\
\hline
\end{tabular}

System Parameters of HD 149026

Notes.-Results are based on fits to the $10^{4}$ Monte Carlo realizations of the data in which $v_{3}$ was dropped (see $\S 4$ ). Values for $M_{*} / M_{\odot}$ were drawn from a Gaussian random distribution with mean 1.30 and standard deviation 0.1 . Values for $R_{*} / R_{\odot}$ were drawn from a Gaussian random distribution with mean 1.45 and standard deviation 0.1 . All other quantities listed in this table were free parameters in the model. 
TABLE 3

Observed Times of Transit

\begin{tabular}{|c|c|c|c|c|c|}
\hline Event & $\begin{array}{c}T_{c} \\
\text { (HJD) }\end{array}$ & $N_{\text {elapsed }}$ & $\begin{array}{c}\sigma_{\text {HJD }} \\
\text { (days) }\end{array}$ & $\begin{array}{c}(O-C) \\
\text { (days) }\end{array}$ & $(O-C) / \sigma_{\mathrm{HJD}}$ \\
\hline Sato et al. (2005) & 2453504.8689 & -8.0 & 0.0019 & +0.0022 & +1.15 \\
\hline Sato et al. (2005) & 2453527.8727 & 0.0 & 0.0017 & -0.0018 & -1.09 \\
\hline This work (FLWO48 $g$ ) …….................. & 2453527.8728 & 0.0 & 0.0012 & -0.0018 & -1.41 \\
\hline Sato et al. (2005) & 2453530.7503 & +1.0 & 0.0012 & -0.0002 & -0.17 \\
\hline 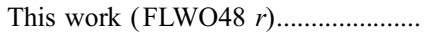 & 2453553.7587 & +9.0 & 0.0010 & +0.0003 & +0.32 \\
\hline This work (TopHAT $V$ ) & 2453553.7597 & +9.0 & 0.0023 & +0.0012 & +0.51 \\
\hline
\end{tabular}

The one-dimensional probability distribution for $i$ shows that the majority of solutions favor $i \approx 86^{\circ}$, but a peak is evident at $i=90^{\circ}$ (see the bottom left panel of Fig. 2). We identify these maximum-i solutions by applying an arbitrary cutoff of $i>89^{\circ} .75$, and we display these solutions with a distinct coloring in Figure 3. These solutions, which account for $9 \%$ of the total, result from Monte Carlo runs in which the value of the stellar radius (which is drawn from a Gaussian distribution; $\S 3$ ) is significantly smaller than the central value of $1.45 R_{\odot}$. For these cases, an edge-on inclination is preferred so that the duration of the model transit is as long as possible, in order to match that of the observations. We exclude this solution subset prior to determining the bestfit values and confidence limits listed in Table 2 . We note that the duration of ingress and egress is significantly shorter for HD 149026 than for either HD 209458 or TrES-1. The available photometry samples the times of ingress and egress only sparsely. We encourage high-cadence monitoring of these key portions of the transit curve.

Based on this analysis, our best estimate of the transit ephemeris is

$$
T_{c}=\left(2,453,527.87455_{-0.00091}^{+0.00085}\right)+\left(2.87598_{-0.00017}^{+0.00012}\right) N(\mathrm{HJD}) .
$$

Tabulation of the observed time of each eclipse is of interest because deviations from the predictions of a single-period ephemeris could indicate the presence of planetary satellites or additional planetary companions (Brown et al. 2001; Miralda-

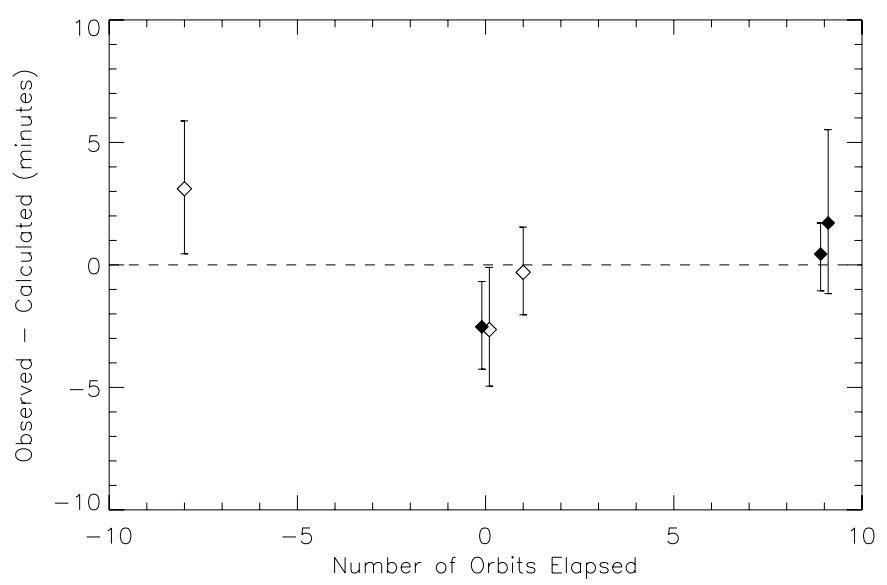

FIG. 4.- "Observed minus calculated" $(O-C)$ residuals for the three light curves presented in this paper ( filled diamonds) and for the three light curves in Sato et al. (2005; open diamonds). In the two cases where two estimates are available for the same transit, we have slightly offset the values along the horizontal axis for clarity. The assumed ephemeris is given in eq. (5). The data plotted here are given in Table 3.
Escudé 2002). In particular, terrestrial planets that induce a radial-velocity perturbation below current detection limits can nonetheless be detected through accurate eclipse timing (Holman \& Murray 2005; Agol et al. 2005). We searched for evidence of timing anomalies as follows. We constructed a model transit light curve for each of the four bandpasses [ $g, r, V$, and $(b+y) / 2]$, based on the optimal parameters appearing in Table 2 and the appropriate limb-darkening coefficient $(\S 3)$. For each of the six extinction-corrected, normalized light curves (identified by an index $j$ ), we then evaluated the $\chi^{2}$ of the fit as a function of assumed time of the center of eclipse, $T_{c}^{j}$. After we identified the optimal value for $T_{c}^{j}$, we assigned uncertainties by identifying the timing offsets at which the value of $\chi^{2}$ had increased by 1 . We list these values in Table 3 and plot the "observed minus calculated" $(O-C)$ residuals in Figure 4. The typical timing precision is 2 minutes, which is comparable to results from other groundbased photometry (for a tabulation of $O-C$ for other transitingplanet systems, see Charbonneau et al. [2005] for TrES-1, and Wittenmyer et al. [2005] for HD 209458). We find no evidence for deviations from the predictions of a constant orbital period. We encourage future monitoring of the times of eclipse of this system. In particular, space-based observations (Brown et al. 2001) should achieve a substantial improvement in timing precision and thus permit a more sensitive search for perturbing planets.

Due to its favorable apparent brightness, the HD 149026 system will be particularly amenable to a variety of follow-up studies. Such pursuits will be more observationally challenging than was the case for HD 209458, owing to the smaller size of the planet relative to the parent star. Nonetheless, we are certain that such challenges will be met and overcome. Now that a handful of transiting planets of bright stars have been identified, the longsought-after goal of comparative exoplanetology may be realized.

We are very grateful to B. Sato, D. Fischer, G. Laughlin, and the other members of the N2K consortium for sharing their results in advance of publication. We thank T. Spahr, R. Kirshner, and M. Hicken for swapping telescope nights on short notice, and $\mathrm{H}$. Knutson for assistance with the remote observations. We thank T. Brown and S. Gaudi for comments that improved the manuscript. Some of the Minicam observations on the FLWO 48 inch telescope were obtained and reduced with support from the Kepler Mission to SAO and PSI. The TopHAT observations were supported by NASA grant NNG 04-GN74G. Work by J. N. W. was supported by NASA through grant HST-HF-01180.02-A, and work by G. B. was supported by NASA through grant HSTHF-01170.01-A, awarded by the Space Telescope Science Institute, which is operated by the Association of Universities for Research in Astronomy, Inc., for NASA, under contract NAS 5-26555. 
Agol, E., Steffen, J., Sari, R., \& Clarkson, W. 2005, MNRAS, 359, 567

Alonso, R., et al. 2004, ApJ, 613, L153

Bakos, G., Noyes, R. W., Kovács, G., Stanek, K. Z., Sasselov, D. D., \& Domsa, I. 2004, PASP, 116, 266

Bouchy, F., Pont, F., Santos, N. C., Melo, C., Mayor, M., Queloz, D., \& Udry, S. 2004, A\&A, 421, L13

Brown, T. M. 2003, ApJ, 593, L125

Brown, T. M., Charbonneau, D., Gilliland, R. L., Noyes, R. W., \& Burrows, A. 2001, ApJ, 552, 699

Brown, T. M., Libbrecht, K. G., \& Charbonneau, D. 2002, PASP, 114, 826

Bundy, K. A., \& Marcy, G. W. 2000, PASP, 112, 1421

Charbonneau, D. 2004, in IAU Symp. 219, Stars as Suns: Activity, Evolution and Planets, ed. A. K. Dupree \& A. O. Benz (San Francisco: ASP), 367

Charbonneau, D., Brown, T. M., Dunham, E. W., Latham, D. W., Looper, D. L., \& Mandushev, G. 2004, in AIP Conf. Proc. 713, The Search for Other Worlds, ed. S. S. Holt, F. W. Olin, \& D. Deming (New York: AIP), 151

Charbonneau, D., Brown, T. M., Latham, D. W., \& Mayor, M. 2000, ApJ, 529, L45

Charbonneau, D., Brown, T. M., Noyes, R. W., \& Gilliland, R. L. 2002, ApJ, 568,377

Charbonneau, D., et al. 2005, ApJ, 626, 523

Claret, A. 2004, A\&A, 428, 1001

Claret, A., \& Hauschildt, P. H. 2003, A\&A, 412, 241

Cody, A. M., \& Sasselov, D. D. 2002, ApJ, 569, 451

Deming, D., Brown, T. M., Charbonneau, D., Harrington, J., \& Richardson, L. J. 2005a, ApJ, 622, 1149

Deming, D., Seager, S., Richardson, L. J., \& Harrington, J. 2005b, Nature, 434, 740

Dravins, D., Lindegren, L., Mezey, E., \& Young, A. T. 1998, PASP, 110, 610

Henry, G. W., Marcy, G. W., Butler, R. P., \& Vogt, S. S. 2000, ApJ, 529, L41

Holman, M. J., \& Murray, N. W. 2005, Science, 307, 1288

Holman, M. J., Winn, J. N., Stanek, K. Z., Torres, G., Sasselov, D. D., Allen, R. L., \& Fraser, W. 2006, ApJ, submitted (astro-ph/0506569)

Konacki, M., Torres, G., Jha, S., \& Sasselov, D. D. 2003, Nature, 421, 507

Konacki, M., Torres, G., Sasselov, D. D., \& Jha, S. 2005, ApJ, 624, 372

Kotredes, L., Charbonneau, D., Looper, D. L., \& O'Donovan, F. T. 2004, in AIP Conf. Proc. 713, The Search for Other Worlds, ed. S. S. Holt, F. W. Olin, \& D. Deming (New York: AIP), 173

\section{EFERENCES}

Latham, D. W. 2003, in ASP Conf. Ser. 294, Scientific Frontiers in Research on Extrasolar Planets, ed. D. Deming \& S. Seager (San Francisco: ASP), 409

Mandel, K., \& Agol, E. 2002, ApJ, 580, L171

Mandushev, G., et al. 2005, ApJ, 621, 1061

Miralda-Escudé, J. 2002, ApJ, 564, 1019

Moutou, C., Coustenis, A., Schneider, J., Queloz, D., \& Mayor, M. 2003, A\&A, 405,341

Moutou, C., Coustenis, A., Schneider, J., St Gilles, R., Mayor, M., Queloz, D., \& Kaufer, A. 2001, A\&A, 371, 260

Moutou, C., Pont, F., Bouchy, F., \& Mayor, M. 2004, A\&A, 424, L31

Narita, N., et al. 2005, PASJ, 57, 471

Ohta, Y., Taruya, A., \& Suto, Y. 2005, ApJ, 622, 1118

Pont, F., Bouchy, F., Queloz, D., Santos, N. C., Melo, C., Mayor, M., \& Udry, S. 2004, A\&A, 426, L15

Press, W. H., Teukolsky, S. A., Vetterling, W. T., \& Flannery, B. P. 1992, in Numerical Recipes in C (Cambridge: Cambridge Univ. Press), 408

Queloz, D., Eggenberger, A., Mayor, M., Perrier, C., Beuzit, J. L., Naef, D., Sivan, J. P., \& Udry, S. 2000, A\&A, 359, L13

Richardson, L. J., Deming, D., \& Seager, S. 2003a, ApJ, 597, 581

Richardson, L. J., Deming, D., Wiedemann, G., Goukenleuque, C., Steyert, D., Harrington, J., \& Esposito, L. W. 2003b, ApJ, 584, 1053

Sato, B., et al. 2005, ApJ, 633, 465

Sozzetti, A., et al. 2004, ApJ, 616, L167

Stetson, P. B. 1994, PASP, 106, 250

Torres, G., Konacki, M., Sasselov, D. D., \& Jha, S. 2004, ApJ, 614, 979

Udalski, A., Szewczyk, O., Zebrun, K., Pietrzynski, G., Szymanski, M., Kubiak, M., Soszynski, I., \& Wyrzykowski, L. 2002, Acta Astron., 52, 317 Udalski, A., Szymanski, M. K., Kubiak, M., Pietrzynski, G., Soszynski, I., Zebrun, K., Szewczyk, O., \& Wyrzykowski, L. 2004, Acta Astron., 54, 313 Vidal-Madjar, A., Lecavelier des Etangs, A., Désert, J.-M., Ballester, G. E., Ferlet, R., Hébrard, G., \& Mayor, M. 2003, Nature, 422, 143

Vidal-Madjar, A., et al. 2004, ApJ, 604, L69

Winn, J. N., Suto, Y., Turner, E. L., Narita, N., Frye, B., Aoki, W., Sato, B., \& Yamada, T. 2004, PASJ, 56, 655

Winn, J. N., et al. 2005, ApJ, 631, 1215

Wittenmyer, R. A., et al. 2005, ApJ, 632, 1157 